5 Authors: Kirsty L. Nash, Christopher Cvitanovic, Elizabeth A. Fulton, Benjamin S.

\section{Planetary Boundaries for a Blue Planet}

Halpern, E.J. Milner-Gulland, Reg A. Watson, Julia L. Blanchard

\section{Supplementary Note 1: Glossary}

Supplementary Note 2: Literature review methods

Supplementary Note 3: Materials and methods for calculating and mapping human appropriated net primary production, including data availability statement

Supplementary Table 1: Trophic efficiency schedule detailing percentage energy transfer among trophic levels

Supplementary Figure 1: Publications referencing planetary boundaries

\section{References}

